DOI: $10.15593 / 2224-9982 / 2017.49 .06$

УДК 629.7.036.3

\title{
В.Г. Августинович
}

Пермский национальный исследовательский политехнический университет, Пермь, Россия

\section{ОСРЕДНЕНИЕ НЕСТАЦИОНАРНЫХ НЕОДНОРОДНЫХ ПОТОКОВ В ТУРБОМАШИНАХ ДЛЯ ОЦЕНКИ ИХ КПД}

\begin{abstract}
Рассматривается подход к осреднению нестационарного неоднородного по пространству потока, основанный на использовании всех четырех уравнений сохранения (массы, энергии, импульса и энтропии). В качестве четвертой независимой переменной наряду с полным и статическим давлением и полной температурой используется измеренная пульсация скорости потока, квадрат которой, осредненный по времени, осредняется затем по расходу с использованием уравнения сохранения потока энтропии. В результате система всех четырех уравнений сохранения становится замкнутой. На основании численного моделирования нестационарного потока в межлопаточном канале турбины и в осевом зазоре между сопловым аппаратом и рабочим колесом показаны особенности генерации и конвекции потока энтропии и существенное влияние нестационарности на формирование граничных условий вниз по потоку, а следовательно, и на оценку КПД. Генерация потока энтропии была получена с помощью метода прямого численного моделирования.

Ключевые слова: турбомашины, КПД турбомашин, осреднение параметров газового потока, нестационарное течение, пульсации скорости, пространственная неоднородность потока, численное моделирование течения в турбине, генерация потока энтропии.
\end{abstract}

\section{V.G. Avgustinovich}

Perm National Research Polytechnic University, Perm, Russian Federation

\section{AVERAGING UNSTEADY NON-UNIFORM FLOW IN TURBOMASHINES FOR EFFICIENCY ESTIMATION}

\begin{abstract}
Approach to averaging of unsteady non-uniform flow based on all four conservation equations (mass, energy, enthalpy, enthropy) using is presented. Fourth independent variable quantity (together with total and static pressure, total temperature) is measured and averaged on time velocity pulsation square. Velocity pulsation square is averaged further on mass flow proceeding from enthropy flow conservation. In result all four conservation equations system is closed. Peculiarities of enthropy flow generating and convection in turbine blade passage on base numerical simulation are presented. Enthropy generation is valued with using of direct numerical simulation procedure. The unsteady influence on confine conditions is also presented.

Keywords: turbomashine, efficiency, flow parameters averaging, unsteady flow, velocity pulsations, non-uniform flow, numerical simulation of flow in turbomashines, enthropy flow generation.
\end{abstract}

При экспериментальном определении коэффициентов полезного действия (КПД) турбомашин и газотурбинных двигателей традиционно используется методика осреднения неоднородных потоков, основанная на приведении реального трехмерного нестационарного потока к одномерному на базе сохранения термодинамических свойств реального потока (потоков массы, энтальпии, количества движения-импульса и энтропии). Это обусловлено тем, что принято оценивать эффективность преобразования теплосодержания в механическую работу одним числом (КПД), т.е. интегрально. Как правило, мощность на валу турбомашины не измеряется, тем более в системе ГТД. В связи с этим производимая работа и располагаемая (идеальная) работа, отношение которых и есть КПД, определяются по термодинамическим параметрам на входе и выходе в предположении определенности вида процесса (адиабатический и т.п.). Сложность такого метода заключается в том, что параметры потока неоднородны по площади проходных сечений, т.е. требуют осреднения. Кроме того, при экспериментальном определении КПД турбомашины на стенде нужно иметь в виду, что граничные условия на установке и в сис- 
теме ГТД различны (например, степень завихренности потока). Это, в свою очередь, влияет на оценку КПД по данной методике осреднения параметров и должно учитываться при переносе оценок КПД на другие граничные условия. Наконец, поток в турбомашинах существенно нестационарный, и приведение его к стационарному тоже вызывает проблему.

Поскольку одномерный стационарный поток полностью описывается тремя параметрами (давление, температура, скорость), то для выполнения условия сохранения используются соответственно три (из четырех) интеграла. В связи с этим возникает проблема выбора набора трех свойств потока из четырех для осреднения неоднородых потоков. При этом четвертое соотношение сохранения интегрального свойства потока не выдерживается, т.е. сама задача сведения неоднородного по пространству потока к одномерному является некорректной (часть свойств теряется). Тем не менее существующая методика оценки КПД ГТД с помощью приведения к одномерной модели течения является общепринятой. На эту тему (выбора оптимального набора «три из четырех» по критерию минимальных погрешностей в оценке КПД) существует большой корпус литературы. В качестве примеров укажем на наиболее фундаментальные работы Л.И. Седова, Э. Грейцера $[1,2]$ и др. [3-8], появившиеся в течение 50 лет.

Какова же относительно недавняя по времени рекомендация Грейцера по решению этой проблемы? Он предлагает принимать решение о выборе «три из четырех» «в контексте проблемы интересов» [2, с. 256], выделяя в качестве приоритетных адекватно осредненные расход газа (уравнение сохранения массы) и полную энтальпию (уравнение сохранения энергии) как главные параметры, определяющие мощность турбомашины. В связи с этим, по сути, проблема возникает при выборе уравнения сохранения для осреднения полного давления между уравнениями сохранения импульса, энтропии или изоэнтропической работы. Именно здесь возникает «проблема интересов». В случае применения уравнения сохранения импульсов мы получаем дополнительные потери за счет необратимости при «смешении» потоков с различной температурой. Это не всегда соответствует реальности, так как реальное смешение требует определенной длины перемешивания, что для компактных конструкций ГТД нехарактерно. В случае применения уравнения сохранения энтропии при осреднении потоков с различной температурой мы получаем нефизичный прирост осредненного полного давления. Наконец, при осреднении полного давления по уравнению сохранения изоэнтропической работы [8] мы получаем не всегда адекватные граничные условия на входе в следующий узел ГТД (каскад компрессора или турбины). Ниже представлены выражения для осреднения полного давления для перечисленных трех способов осреднения:

1) по уравнению расхода

$$
p_{0}=\frac{G \sqrt{\bar{T}_{0}}}{m F q(\bar{\lambda})},
$$

где средняя характеристическая скорость потока $\bar{\lambda}$ определяется из условия сохранения импульса в осредненном и неоднородном потоках (с точностью до постоянства показателя адиабаты), т.е. $\Phi=\sum d \Phi$ :

$$
G \sqrt{\bar{T}_{0}} z(\bar{\lambda})=\int \sqrt{T_{0}} z(\lambda) d G
$$

2) по уравнению сохранения энтропии

$$
\bar{p}_{0}=\left(G \bar{T}_{0} \int \frac{p_{0}^{\frac{k-1}{k}}}{T_{0}} d G\right)^{\frac{k}{k-1}}
$$


3) по уравнению сохранения изоэнтропической работы

$$
\bar{p}_{0}=\left[\frac{G \bar{T}_{0}}{\int_{p_{0} \frac{T_{0}}{k-1} d G}^{k}}\right]^{\frac{k}{k-1}} .
$$

В русле рекомендации Грейцера недавно была опубликована работа В.М. Кофмана [9], обобщающая различные способы осреднения стационарных неоднородных потоков на примерах обработки результатов испытаний различных узлов ГТД.

Следует признать, однако, что такая постановка чрезмерно идеализирована и, соответственно, формирует поле для произвольных оценок КПД узлов ГТД, не говоря о проблематичности идентификации локальных областей генерации потерь и выдаче рекомендаций по их уменьшению с помощью конструктивных мероприятий. Действительно, как правило, основными источниками потерь кинетической энергии потока являются силы вязкости, генерируемые в областях течения с поперечными градиентами скорости (концевые области). Вихревой след является заключительной фазой истории формирования и развития пограничного слоя. Вследствие этого возникают пульсации скорости (и давления) и существенная нестационарность потока, т.е. искомые потери связаны с нестационарностью неразрывно.

Возникающая нестационарность, а также связанная с этим завихренность потока существенно усложняют задачу приведения неоднородного нестационарного потока к одномерному стационарному (осредненному) потоку. В результате осреднение потока в рамках существующей парадигмы оценки КПД по осреднению только пространственной неоднородности потока требует уточнения, особенно в связи с существующей тенденцией повышения нагрузок на ступень и, соответственно, повышения уровня нестационарности.

В термодинамике необратимых процессов вводится понятие диссипативной функции $\psi=T d S / d t$, характеризующей порождение энтропии во времени [10]. Эта функция является единственной научно обоснованной мерой необратимости нестационарных процессов. Изменение энтропии во времени определяется из уравнения баланса энтропии за вычетом потока энтропии по ограничивающей систему поверхности. Этот баланс представляется в виде суммы произведений обобщенных потоков и обобщенных (термодинамических) сил, т.е.

$$
\frac{d s}{d t}=\sum I_{i} X_{i}
$$

Обобщенные силы здесь - это градиенты параметров по координатам. В равновесном состоянии обобщенные потоки и обобщенные силы равны нулю.

Для течения вязкой жидкости (при отсутствии потоков тепла, диффузии, реагирующих компонентов химических реакций) обобщенный поток энтропии является необратимой (вязкой) составляющей потока импульса. В этом случае порождение энтропии во времени приобретает вид

$$
\frac{d s}{d t}=\tau_{i j} \frac{d u_{i}}{d x_{j}}
$$

где $\tau_{i j}$ - тензор вязких, или рейнольдсовых, напряжений, пропорциональный скорости; $\frac{d u_{i}}{d x_{j}}-$ обобщенная сила, пропорциональная скорости.

Это позволяет нам использовать уравнение сохранения потока энтропии для реального и приведенного к однородному одномерному (плоскому) потоков при наличии неоднородности измеренных по сечению пульсаций скорости. 
Таким образом, наряду с давлением, температурой и скоростью потока пульсации скорости должны быть включены в перечень определяющих параметров одномерного потока при определении потерь кинетической энергии. Соответственно, для нахождения осредненных параметров потока должны быть использованы все четыре уравнения сохранения: потоков массы, энергии, импульса и энтропии. В этом случае задача становится корректной, так как для определения четырех неизвестных (параметры осредненного потока) используются четыре уравнения сохранения.

Запишем уравнение импульсов в векторной форме для потока с пульсациями скорости:

$$
\boldsymbol{\Phi}=\rho\left(\mathbf{c} \cdot \mathbf{c}+\tau_{i j}\right) F+p F,
$$

где $\mathbf{c}-$ вектор скорости с компонентами по координатам, т.е. $\mathbf{c}=(u, v, w)^{\mathrm{T}} ; \tau_{i j}-$ тензор вязких напряжений.

Компоненты скорости представляются в виде суммы осредненной во времени скорости и пульсации скорости: $\mathbf{u}=\bar{u}+u^{\prime}, \mathbf{v}=\bar{v}+v^{\prime}, \mathbf{w}=\bar{w}+w^{\prime}$.

Для плоского одномерного потока $\bar{u} \neq 0, \quad u^{\prime} \neq 0, \quad \bar{v}=0, \quad v^{\prime} \neq 0, \quad \bar{w}=0, \quad w^{\prime}=0$. Соответственно, $\tau_{i j}=\left(\overline{u^{\prime 2}},-\overline{u^{\prime} v^{\prime}}\right)^{T}$.

Для иллюстрации существенно пульсационной и завихренной картины течения в межлопаточных каналах турбины на рис. 1 представлены данные математического моделирования нестационарного вязкого течения, полученные методом прямого численного моделирования (DNS - Direct Numerical Simulation) [11].
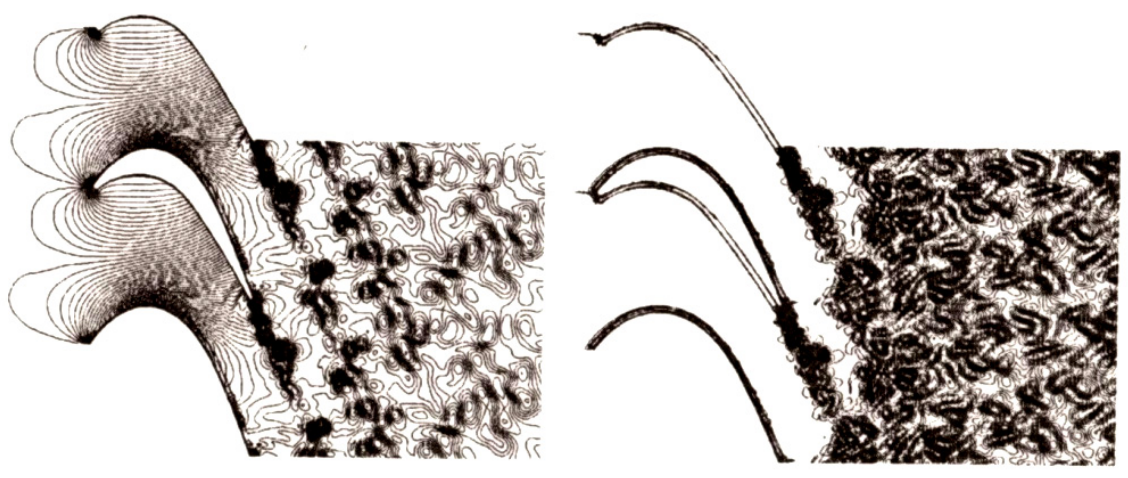

Рис. 1. Мгновенное поле скоростей и энтропии на выходе из турбинной решетки

Как видно из рисунка, в межлопаточных каналах по мере перехода от венца к венцу вихревые структуры занимают всё большую площадь проходного сечения. Здесь же возникает проблема оценки возврата тепла по ступеням в многоступенчатой турбомашине в результате последующей диссипации пульсаций скорости и превращения их в тепло. При измерении температуры торможения потока на выходе из ступени диссипация пульсаций происходит при торможении потока вблизи приемника термопары, в то время как в реальном потоке вихревые структуры, генерирующие пульсации скорости, устойчиво конвектируют к выходу. Таким образом, при использовании измеренной полной температуры на выходе из ступени мы получаем адекватную оценку КПД ступени, но завышаем температуру на входе в следующую ступень с последующей погрешностью оценки ее КПД (или расположенной вслед за турбиной высокого давления, например, турбины среднего или низкого давления). Практикуемое кильватерное расположение сопловых лопаток («след в след», или clocking) позволяет получить возврат тепла за счет диссипации пульсаций скорости в границах турбомашины (на следующей ступени) и тем самым повысить ее КПД. 
Мощным источником нестационарности и завихренности в ГТД является камера сгорания. Это обусловлено необходимостью интенсификации и обеспечения устойчивости процесса горения. Для этого во фронтовом устройстве (горелке) наводится турбулентность для повышения скорости горения и поперечного перемешивания и формируется центральный вихрь, обеспечивающий устойчивость горения за счет объемной зоны обратных токов горячих продуктов сгорания по центру вихря. При этом вихревое ядро прецессирует, генерируя регулярные пульсации скорости. Наконец, обтекание струй холодного воздуха, подаваемых в зону смешения камеры сгорания, также сопровождается интенсивным вихреобразованием. На выходе из камеры сгорания (входе в турбину) стохастические пульсации скорости (интенсивность турбулентности) достигают $35 \%$, а на выходе из соплового аппарата - 65 \% [12]. При этом следует иметь в виду, что такая высокая интенсивность турбулентности реализуется при отнесении пульсации скорости к пиковому значению скорости течения на выходе из межлопаточного канала турбинной решетки.

Вместе с этим при транспортировке через межлопаточный канал соплового аппарата вихревые структуры конвектируют в области минимального давления (спинка лопатки), занимая достаточно большую площадь проходного сечения. Диапазон пульсаций скорости (интенсивности турбулентности), как показывают эксперименты и математическое моделирование с применением LES-технологии (Large Eddy Simulation), составляет 10-65 \% [12]. При этом интенсивность турбулентности распределяется неравномерно по сечению, а вихревые жгуты, формирующиеся в камере сгорания, относятся к классу устойчивых и долгоживущих. Растягиваясь в маршевом направлении и уменьшаясь в масштабе за счет увеличения скорости в межлопаточном канале турбины, эти вихревые структуры не только увеличивают интенсивность турбулентности (в данном эксперименте от 35 до $65 \%$ ), но и приобретают анизотропную структуру, обесценивая применение модели турбулентности типа $k-\varepsilon$ в RANS (Reynolds Averaged Navier Stokes) CFD-подходе.

Кроме случайных пульсаций, генерируемых силами молекулярной и турбулентной вязкости, в лопаточных машинах генерируются и регулярные пульсации давления-скорости в результате потенциального взаимодействия движущейся и неподвижной решеток профилей. В качестве примера регулярной пульсации скорости (давления) потока в ступени турбины на рис. 2 представлено изменение скорости и давления в фазовых координатах (скоростьдавление), полученное в результате математического моделирования статор-ротор-взаимодействия на конкретной турбине [11]. Здесь каждой точке графика соответствует свой момент времени, который не отражен на рисунке, чтобы не загромождать его. Очевидно, что в силу периодичности прохождения лопатки рабочего колеса в потенциальном поле взаимодействия с неподвижной лопаткой соплового аппарата фазовая траектория имеет замкнутый вид. Следует отметить, что в данной (эйлеровой) постановке процесс в осевом зазоре между ротором и статором моделировался методом Ю.М. Давыдова (МКЧ - метод крупных частиц) без обычно применяемого в практике осреднения параметров по скользящему интерфейсу, т.е. адекватно.

Как видно из рис. 2, в результате статор-ротор-взаимодействия наблюдается существенный уровень регулярных пульсаций скорости (двойной амплитуды до $10 \%$ ) всего потока. Одним из методов осреднения параметров во времени в этом случае является представление регулярных пульсаций как источника так называемых «детерминистских напряжений» по типу напряжений Рейнольдса, применяемых при осреднении параметров турбулентных потоков. Как показывают исследования [13], такой подход позволяет заметно повысить точность оценки осредненных параметров в турбомашинах. Из рис. 2 видно также, что осредненная за цикл прохождения лопаткой турбины шага соплового аппарата работа расширения-сжатия не равна нулю, а именно имеет отрицательный знак, т.е. работа сжатия больше работы расширения за счет несимметричной геометрии взаимодействующих профилей лопаток статора и ротора. Согласно исследованию [14], эта работа имеет акустическую форму, т.е. статор-ротор-взаимодействие генерирует тоновый шум на «лопаточной» частоте. Эта акустическая работа с точки зрения 
производства механической работы турбины является бесполезной и уменьшает ее КПД. Причем, как видно из рисунка, акустическая работа зависит от величины окружной неоднородности температурного поля на входе в турбину.

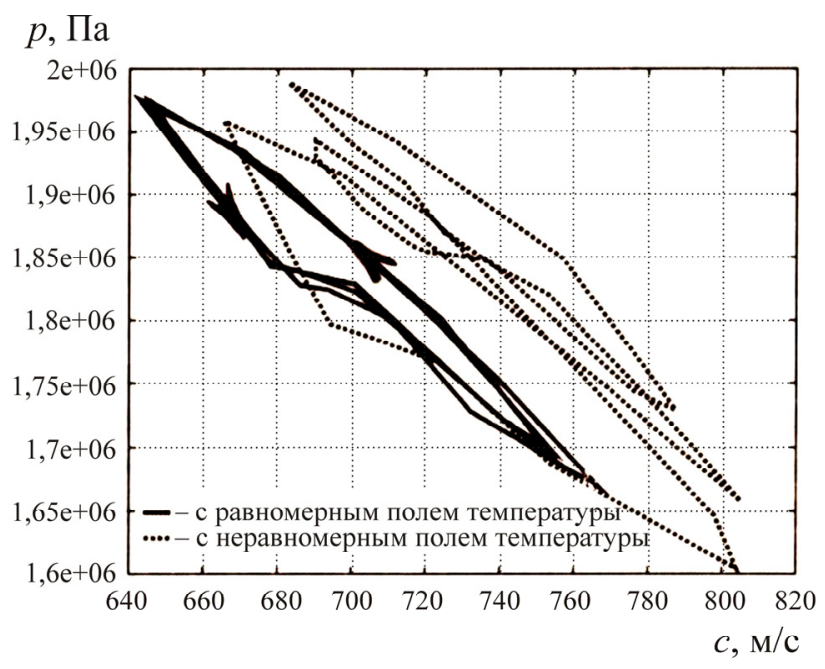

Рис. 2. Фазовая траектория изменения скорости-давления в осевом зазоре между статором и ротором в турбине

Одним из информативных уравнений процессов в ступени турбомашины является уравнение движения (энергии) газа в форме Крокко [15]:

$$
\frac{\mathrm{d} v}{\mathrm{~d} t}+\nabla i_{0}-T d s=\mathbf{v} \times \omega
$$

Как видно из уравнения, реальному потоку присущи такие интегральные составляющие, как нестационарность и завихренность. Нестационарность характеризуется уровнем пульсаций скорости (стохастических и регулярных). В случае стационарного незавихренного изоэнтропического потока мы получаем известное соотношение постоянства полной энтальпии.

Как уже было отмечено выше, для корректного подхода к приведению нестационарного неоднородного потока к стационарному одномерному потоку, модель которого используется при оценках КПД узлов ГТД, необходимо измерять, кроме давления, температуры и скорости (или ее аналога), дополнительно пульсации скорости. Тогда методика обработки экспериметальных данных должна выглядеть следующим образом, исходя из сохранения набора интегральных термодинамических свойств потока $\left(G, I_{0}, S, \Phi\right)$ для реального и приведенного к одномерному стационарному потоков:

1. Производится осреднение по времени измеряемых параметров потока $p, p_{0}, T_{0}, u^{\prime 2}$ (давление - статическое и полное, полная температура и квадрат пульсации скорости в общем случае по трем компонентам, т.е. через определение кинетической энергии турбулентности) по многоточечным приемникам-гребенкам. В типичном случае потоков в ГТД компонентами скорости, кроме маршевого направления потока массы, можно пренебречь.

2. Определяются местные осредненные плотности тока по осредненным по времени параметрам $\bar{p}$ и $\bar{p}_{0}$ (посредством газодинамической функции $\pi(\bar{\lambda})$ ). Здесь же по известным соотношениям для изоэнтропического потока определяются местные статическая температура, плотность и скорость потока.

3. Определяются местные осредненные расходы газа $(d G)$ по осредненной по времени плотности тока и площади условной «струйки тока» $d F$.

4. Определяется суммарный расход газа

$$
G=\sum d G \quad\left(F=\sum d F\right) .
$$


5. Определяется осредненная по расходу полная температура газа из условия сохранения полной энтальпии:

$$
\bar{T}_{0}=\left(1 / c_{p} G\right) \sum i_{0} d G
$$

6. Определяется осредненная по расходу пульсационная составляющая импульса потока $\Phi^{\prime}$ (аналог тензора вязких напряжений) из условия сохранения потока энтропии, а именно

$$
\overline{U^{\prime 2}}=\frac{1}{G} \sum \overline{u^{\prime 2}} d G
$$

7. Определяется осредненная по расходу скорость потока из условия сохранения количества движения (импульса) с учетом пульсаций скорости:

$$
\bar{\rho}\left(\overline{U^{2}}+\overline{U^{\prime 2}}\right) F=\sum \rho\left(\overline{u^{2}}+\overline{u^{\prime 2}}\right) d F,
$$

при этом должно выполняться равенство

$$
\bar{\rho} \bar{U} F=G .
$$

8. Определяется осредненное полное давление по расходу и осредненным полной температуре и скорости потока по формуле (1).

Представленная методика осреднения нестационарного неоднородного потока, как мы видим, отличается от традиционной наличием учета осредненной пульсационной составляющей потока импульса. В случае, если эта составляющая не изменяется по пространству, неучет ее в традиционной методике осреднения не приводит к дополнительным погрешностям определения средней скорости потока. Однако, как известно, зоны повышенных пульсаций скорости потока расположены как раз в области генерации потерь (отрывные течения вблизи обтекаемых поверхностей, зоны парных вихрей и их взаимодействия с подковообразными вихрями в концевых сечениях и т.д.) и зонах струйного взаимодействия с основным потоком. Таким образом, при обработке экспериментальных данных с целью уменьшения погрешности осреднения потерь (КПД) необходимо учитывать нестационарную природу потока в ГТД и его узлах. Здесь же мы видим органическое включение условия сохранения энтропии в осредненном потоке в систему приведения неоднородного нестационарного потока к одномерному стационарному.

\section{Библиографический список}

1. Седов Л.И., Черный Г.Г. Об осреднении неравномерных потоков газа в каналах // Теоретическая гидромеханика. - М.: Оборонгиз, 1954. - Вып. 4, № 12. - С. 17-30.

2. Greitzer E.M., Tan C.S., Graf M.B. Internal flow: concepts and application. - Cambridge, Cambridge University Press, 2004. - 736 p.

3. Cumpsty N.A., Horlock J.H. Averaging non-uniform flow for a purpose // Journal of Turbomachinery. -2005 . - Vol. 128, № 1. - P. 120-129.

4. Schoenweitz D., Schnell R. Development and evaluation of performance estimation methodology for fans operating within non-homogeneous inflow // Proc. ASME Turbo expo GT 2016-57095. - Seoul, 2016. - 12 p. MIT, 2015.

5. Lee J. Aerothermodynamics and operation of turbine system under pulsation flow. Master's thesis. -

6. Performance metric for turbine stage under pulsation flow environment / J. Lee, C. Tan, B. Sirakov, C. Wilkins, Im Hong-Sik, M. Babak, D. Tisserant // Proc. ASME Turbo Expo GT 2016-56343. - Seoul, 2016. - 9 p.

7. Miller R.J. Mechanical work potential // Proc. ASME Turbo Expo 2013. - San Antonio, 2013. - 13 p.

8. Jedamski D. Turbine inlet nonuniformities and unsteady mechanisms. Master's Thesis. - MIT, 2015. $-88 \mathrm{p}$.

9. Кофман В.М. Методология и опыт экспериментально-расчетного определения показателей газодинамической эффективности узлов ГТД по параметрам неравномерных воздушных и газовых потоков / Уфим. гос. авиац. техн. ун-т. - Уфа, 2013. - 400 с.

10. Вукалович М.П., Новиков И.И. Термодинамика. - М.: Машиностроение, 1972. - 627 с. 
11. Численное моделирование нестационарных явлений в ГТД / под ред. В.Г. Августиновича и Ю.Н. Шмотина. - М.: Машиностроение, 2005. - 536 с.

12. Turbulence levels are high at combustor-turbine interface / C.M. Cha, P.T. Ireland, P.A. Denman, V. Savarianandam // Proc. ASME Turbo Expo GT2012-69130. - Copenhagen, 2012. - 20 p.

13. Stollenwerk S., Kugeler E. Deterministic stress modelling for multistage compressor flowfield // Proc. ASME Turbo Expo GT2013-94860. - San Antonio, 2013.

14. Раушенбах Б.В. Вибрационное горение: учеб. пособие. - М: Физматлит, 1961. - 500 с.

15. Аэрогидромеханика / Е.Н. Бондарев, В.Т. Дубасов, Ю.А. Рыжов [и др.]. - М.: Машиностроение, 1993. - 608 с.

\section{References}

1. Sedov L.I., Chernyy G.G. Ob osrednenii neravnomernykh potokov gaza v kanalakh. Teoreticheskaya gidromekhanika [On averaging nonuniform gas flows in ducts. Theoretical hydrodynamics]. Moscow, Oborongiz, 1954 , iss. 4 , no. 12 , pp. 17-30.

2. Greitzer E.M., Tan C.S., Graf M.B. Internal flow: concepts and application. Cambridge, Cambridge University Press, 2004, 736 p.

3. Cumpsty N.A., Horlock J.H. Averaging non-uniform flow for a purpose. Journal of Turbomachinery, 2005, vol. 128 , no. 1 , pp. 120-129.

4. Schoenweitz D., Schnell R. Development and evaluation of performance estimation methodology for fans operating within non-homogeneous inflow. Proc. ASME Turbo expo GT 2016-57095, Seoul, South Korea, 2016, 12 p.

5. Lee J. Aerothermodynamics and operation of turbine system under pulsation flow. Master's thesis, MIT, 2015.

6. Lee J., Tan C., Sirakov B., Wilkins C., Hong-Sik Im, Babak M., Tisserant D. Performance metric for turbine stage under pulsation flow environment. Proc. ASME Turbo Expo GT 2016-56343, Seoul, South Korea, 2016, 9 p.

7. Miller R.J. Mechanical work potential. Proc. ASME Turbo Expo 2013, San Antonio, Texas, USA, 2013, $13 \mathrm{p}$

8. Jedamski D. Turbine inlet nonuniformities and unsteady mechanisms. Master's Thesis-MIT, 2015, 88 p.

9. Kofman V.M. Metodologiya i opyt eksperimentalno-raschetnogo opredeleniya pokazateley gazodinamicheskoy effektivnosti uzlov GTD po parametram neravnomernykh vozdushnykh i gazovykh potokov [Methodology and experience of experimental calculating of gas-dynamic parameter efficiency of gas turbine engine components based on parameters of non-uniform air and gas flows]. Ufa, Ufimskiy gosudarstvennyy aviatsionnyy tekhnicheskiy universitet, 2013, 400 p.

10. Vukalovich M.P., Novikov I.I. Termodinamika [Thermodynamics]. Moscow, Mashinostroenie, 1972, $627 \mathrm{p}$.

11. Avgustinovich V.G., Shmotin Yu.N. Chislennoe modelirovanie nestatsionarnykh yavleniy v GTD [Numerical simulation of nonstationary process in GTE]. Moscow, Mashinostroenie, 2005, 536 p.

12. Cha C.M., Ireland P.T. Denman P.A. Savarianandam V. Turbulence levels are high at combustorturbine interface. Proc. ASME Turbo Expo GT2012-69130, Copenhagen, Denmark, 2012, 20 p.

13. Stollenwerk S., Kugeler E. Deterministic stress modelling for multistage compressor flowfield. Proc. ASME Turbo Expo GT2013-94860, San Antonio, Texas, USA, 2013.

14. Raushenbakh B.V. Vibratsionnoe gorenie [Vibrating combustion]. Moscow, GIFML, 1961.

15. Bondarev E.N., Dubasov V.T., Ryzhov Yu.A. et al. Aerogidromekhanika [Aerohydromechanics]. Moscow, Mashinostroenie, 1993, 608 p.

\section{Об авторе}

Августинович Валерий Георгиевич (Пермь, Россия) - доктор технических наук, профессор кафедры «Авиационные двигатели» ФГБОУ ВО ПНИПУ (614990, г. Пермь, Комсомольский пр., д. 29, e-mail: august.valery@yandex.ru).

\section{About the author}

Valery G. Avgustinovich (Perm, Russian Federation) - Doctor of Technical Science, Professor, Department of Aircraft Engines, Perm National Research Polytechnic University (29, Komsomolsky av., Perm, 614990, Russian Federation, e-mail: august.valery@yandex.ru).

Получено 28.04.2017 